\title{
Correlation of Temperature with Platelet Count and Total Leukocyte Count in Dengue: Findings from a Secondary Data Analysis
}

\author{
Abubakr Momin ${ }^{1 *}$, Zarghoona wajid ${ }^{2}$, Munsif Adil ${ }^{3}$, Fareha Haleem ${ }^{4}$, Ahsan Ali Siddiqui ${ }^{5}$, \\ Anam Ahmed ${ }^{6}$, Shahzain Hasan ${ }^{7}$ and Adnan Anwar ${ }^{8}$ \\ ${ }^{1}$ MBBS, Post Graduate trainee, Internal medicine, Medicine Department, Ziauddin hospital \\ ${ }^{2}$ MBBS, M Assistant, Musavvir Stem cell Clinic and Pathology Laboratory, Pakistan \\ ${ }^{3}$ MBBS, Medical Officer, Medicare Hospital Karachi \\ ${ }^{4}$ MBBS, Women Medical Officer, Department of ENT, Civil Hospital, Karachi \\ ${ }^{5}$ MBBS, Msc Public Health, Specialist Family Medicine, Ministry of Health, Riyadh \\ ${ }^{6}$ MBBS, Post Graduate trainee, Internal medicine, Medicine Department, Jinnah Hospital \\ ${ }^{7}$ MBBS, Medical Officer, Hamdard University Hospital, Pakistan \\ ${ }^{8}$ MBBS, M.Phil, Assistant Professor Department of Physiology Al Tibri Medical College Karachi
}

Received: January 16, 2019; Accepted: January 17, 2019 ; Published: February 02, 2019

*Corresponding author: Abubakr Momin, MBBS, Post Graduate trainee, Internal medicine, Medicine Department, Ziauddin hospital. E-mail: abubakr_ss@yahoo.com

\begin{abstract}
Background: Dengue fever annually affects almost 100 million people globally with 2.5 billion people at risk of developing dengue fever. The symptoms of dengue fever begins with flu like illness and may lead to severe manifestations such as bleeding, sudden loss of consciousness and even loss of life. The treatment of the infection is decided on the basis of severity of clinical features and level of fluids in the body.

Objective: To assess the correlation of temperature with platelet count and total leukocyte count in patients of dengue fever.

Methods: A secondary data analysis of a randomized controlled trial on the effects of silymarin on hepatic enzymes and clinical manifestation of dengue fever was carried out including only the patients in the placebo group of the trial. The correlation of temperature with platelet count and total leukocyte count was assessed using Spearman's correlation coefficient. The significance level was set at 0.05 .

Results: The study results revealed that the temperature was significantly negatively correlated with total leukocyte count on day 3 only $(p=0.026)$. Furthermore, in patients with fever duration of 5 to 7 days it was significantly negatively correlated with platelet count on day 3 only $(p=0.002)$ whereas in patients aged 40 years or above it was significantly negatively correlated with total leukocyte count on day 3 only ( $p=0.025)$.

Conclusion: Raised temperature was found to be significantly negatively correlated with total leukocyte count on day 3 only. Moreover, stratified analysis showed it to be significantly negatively correlated with platelet count on day 3 in patients with longer duration of fever and with total leukocyte count on day 3 in patients aged 40 years or above. Further evaluation of the study findings with a larger sample size is recommended.
\end{abstract}

Key words: Correlation, temperature, platelet count, total leukocyte count, dengue, secondary data analysis

\section{Introduction}

A mosquito induced viral infection; dengue fever annually affects almost 100 million people globally with 2.5 billion people at risk of developing dengue fever. [1] It has four viral strains i.e. DEN-1, DEN-2, DEN-3, and DEN-4. [2] Majority of the cases of dengue fever have been estimated to be caused by DEN-2 and DEN-3 viral strains.[3, 4]The symptoms of dengue fever begins with flu like illness and may lead to severe manifestations such as bleeding, sudden loss of consciousness and even loss of life. [5] Fever usually persists for one week while within first two days patients feel warmth and redness on face or neck with red spots under the skin. [6] Its clinical manifestations include headache, fever, skin rashes, leucopenia and arthralgia. Dermatological manifestations occur in almost $50 \%$ of cases and begin just after patient appears to be afebrile. [6, 7]Bleeding occurs more frequently in patients with severe thrombocytopenia. [8]

Dengue fever and dengue hemorrhagic fever are diagnosed on the basis of clinical and epidemiological parameters. The treatment plan depends upon detection of IgM and IgG antibodies in the patient's blood whereas ELISA is used to differentiate between primary and secondary dengue infections. [9] For rapid diagnosis of the dengue virus, NS1 antigens are detected in the 
patient's blood. [10]The treatment of the infection is decided on the basis of severity of clinical features and level of fluids in the body. Patients with hemorrhagic manifestations such as petechiae, gum-bleeding, epistaxis etc. require platelet transfusion. [8]

Dengue is prevented by mosquitoes control and by protecting the subjects from mosquito bite. [11]Viral prevention strategies include properly disposing off waste, up gradation of water storage system and proper usage of repellents. [9] There is no recommended vaccine yet for the eradication of the virus though a new live attenuated tetravalent vaccine is undergoing clinical trials. [12]

To the best of author's knowledge, local data evaluating any potential association of fever with platelet count and total leukocyte count in the context of dengue fever are limited at best. The current study was therefore designed to assess the correlation of temperature with platelet count and total leukocyte count in patients of dengue fever by means of a secondary data analysis.

\section{Methods}

After taking ethical approval, a secondary data analysis of a randomized controlled trial on the effects of silymarin on hepatic enzymes and clinical manifestation of dengue fever was carried out. The trial was conducted at Ziauddin Hospital, Clifton, and Karachi. For the sake of current study, only the patients in the placebo group of the trial $(n=46)$ were included in the analysis These patients, aged 18 to 70 years, had been diagnosed clinically with a positive serological test. The relevant data were analyzed on SPSS version 20. After checking normality, the correlation of temperature with platelet count and total leukocyte count was assessed using Spearman's correlation coefficient. The significance level was set at 0.05 .

\section{Results}

In a total of 46 patients the mean level of platelet count at $1^{\text {st }}, 2^{\text {nd }} 3^{\text {rd }}, 4^{\text {th }}$ and $5^{\text {th }}$ day was observed to be $82.28 \pm 44.62 \times 10^{3}$, $74.45 \pm 37.7 \times 10^{3}, 77.53 \pm 43.12 \times 10^{3}$, and $93.59 \pm 58.69 \times 10^{3}$, $99.0 \pm 77.11 \times 10^{3}$ cells/cumm respectively. Similarly the mean of leukocyte count was observed to be $3.7 \pm 1.62 \times 103,4.4 \pm 2.69$ $\times 10^{3}, 5.53 \pm 3.02 \times 10^{3}$, and $5.97 \pm 3.33 \times 10^{3}, 6.25 \pm 3.06 \times 10^{3}$ cells/cumm respectively. The study results revealed that the temperature was not significantly correlated with platelet count whereas it was significantly correlated with total leukocyte count on day 3 only $(\rho=-0.329, p=0.026)$ (table 1$)$.

The study results revealed that in patients with fever duration of up to 4 days the temperature was not significantly correlated with either platelet count or total leukocyte whereas in patients with fever duration of 5 to 7 days it was significantly correlated with platelet count on day 3 only $(\rho=-0.681, p=0.002)$ (table 2$)$.

The study results also showed that in patients aged $<40$ years the temperature was not significantly correlated with either platelet count or total leukocyte whereas in patients aged 40 years or above it was significantly correlated with total leukocyte count on day 3 only $(\rho=-0.576, p=0.025)$ (table 3 ).

Table 1: Correlation of Temperature with Platelet Count and Total Leukocyte Count

\begin{tabular}{|c|c|c|c|c|}
\hline Table 1: Correlation of Temperature with Platelet Count and Total Leukocyte Count \\
\hline \multirow{2}{*}{ Variables (n=46) } & Platelet Count & $\mathbf{p}$ & \multicolumn{2}{c|}{ Total Leukocyte Count } \\
\cline { 2 - 5 } & $\mathbf{P}$ & 0.331 & 0.068 & $\mathbf{\rho}$ \\
\hline Day 1 Temperature & -0.146 & 0.246 & -0.199 & 0.654 \\
\hline Day 2 Temperature & -0.174 & 0.239 & -0.329 & 0.026 \\
\hline Day 3 Temperature & -0.177 & 0.314 & -0.279 & 0.061 \\
\hline Day 4 Temperature & -0.152 & 0.643 & 0.023 & 0.881 \\
\hline Day 5 Temperature & -0.070 & & P \\
\hline
\end{tabular}

Table 2: Correlation of Temperature with Platelet Count and Total Leukocyte Count Stratified for Fever Duration

\begin{tabular}{|l|c|c|c|c|c|c|c|c|}
\hline \multirow{2}{*}{ Variables } & \multicolumn{4}{|c|}{ Fever Duration of up to 4 Days (n=28) } & \multicolumn{3}{c|}{ Fever Duration of 5 to 7 Days (n=18) } \\
\cline { 2 - 9 } & \multicolumn{2}{|c|}{ Platelet Count } & Total Leukocyte Count & \multicolumn{2}{c|}{ Platelet Count } & \multicolumn{2}{c|}{ Total Leukocyte Count } \\
\cline { 2 - 9 } & $\mathbf{P}$ & $\mathbf{p}$ & $\mathbf{\rho}$ & $\mathbf{p}$ & $\mathbf{P}$ & $\mathbf{P}$ & $\mathbf{\rho}$ & $\mathbf{p}$ \\
\hline Day 1 Temperature & -0.189 & 0.335 & 0.087 & 0.659 & 0.096 & 0.704 & 0.097 & 0.701 \\
\hline Day 2 Temperature & -0.211 & 0.28 & -0.134 & 0.497 & -0.092 & 0.716 & -0.296 & 0.233 \\
\hline Day 3 Temperature & 0.118 & 0.548 & -0.343 & 0.074 & -0.681 & $0.002^{*}$ & -0.280 & 0.26 \\
\hline Day 4 Temperature & 0.053 & 0.79 & -0.357 & 0.062 & -0.357 & 0.146 & -0.135 & 0.593 \\
\hline Day 5 Temperature & -0.324 & 0.093 & 0.056 & 0.779 & 0.227 & 0.366 & 0.065 & 0.799 \\
\hline
\end{tabular}




\begin{tabular}{|c|c|c|c|c|c|c|c|c|}
\hline \multirow{3}{*}{ Variables } & \multicolumn{4}{|c|}{ Age $<40$ Years $(n=31)$} & \multicolumn{4}{|c|}{ Age 40 Years or Above $(n=15)$} \\
\hline & \multicolumn{2}{|c|}{ Platelet Count } & \multicolumn{2}{|c|}{ Total Leukocyte Count } & \multicolumn{2}{|c|}{ Platelet Count } & \multicolumn{2}{|c|}{ Total Leukocyte Count } \\
\hline & $\mathbf{P}$ & $\mathbf{p}$ & $\rho$ & $\mathbf{p}$ & $\mathbf{P}$ & $\mathbf{P}$ & $\rho$ & $\mathbf{p}$ \\
\hline Day 1 Temperature & -0.164 & 0.378 & 0.128 & 0.494 & -0.082 & 0.77 & -0.101 & 0.721 \\
\hline Day 2 Temperature & -0.350 & 0.054 & -0.210 & 0.256 & 0.205 & 0.465 & -0.207 & 0.458 \\
\hline Day 3 Temperature & -0.233 & 0.207 & -0.172 & 0.354 & 0.043 & 0.878 & -0.576 & $0.025^{*}$ \\
\hline Day 4 Temperature & -0.119 & 0.523 & -0.228 & 0.218 & -0.182 & 0.517 & -0.341 & 0.214 \\
\hline Day 5 Temperature & -0.086 & 0.644 & 0.062 & 0.74 & $\ldots$. & $\ldots$. & $\ldots$. & $\ldots$ \\
\hline
\end{tabular}

\section{Discussion}

The study results revealed that the temperature was not significantly correlated with platelet count whereas it was significantly negatively correlated with total leukocyte count on day 3 only $(\mathrm{p}<0.05)$. Furthermore, in patients with fever duration of up to 4 days the temperature was not significantly correlated with either platelet count or total leukocyte whereas in patients with fever duration of 5 to 7 days it was significantly negatively correlated with platelet count on day 3 only $(\mathrm{p}<0.05)$. Moreover, in patients aged $<40$ years the temperature was not significantly correlated with either platelet count or total leukocyte whereas in patients aged 40 years or above it was significantly negatively correlated with total leukocyte count on day 3 only $(\mathrm{p}<0.05)$.

As expected, the study results revealed a negative correlation of temperature with the platelet count and total leukocyte count of the dengue patients, albeit only on day 3 which was an interesting finding in itself. Unfortunately, a thorough literature search did not reveal any pertinent published data to make a meaningful comparison of the study results.

In the management of dengue fever, platelet transfusion is considered when patients manifest bleeding problems such as petechiae, gum-bleeding, epistaxis etc. which occur more frequently in patients with severe thrombocytopenia. [8] Moreover, a large cross-sectional study from Pakistan in 2010 reported that while a low total white cell count was seen more commonly in patients with dengue fever as compared to dengue hemorrhagic fever $(p=0.020)$, Neutropenia $(p=0.019)$ and monocytosis $(p=0.001)$ were found to be more common in the latter group. [13]

Literature shows that decisions taken using simple clinical and hematological parameters can predict diagnosis and prognosis of dengue fever in the early phase of illness. [14] If it can be unequivocally established that raised temperature in dengue hemorrhagic fever is inversely correlated with platelet count and total leukocyte count, it could be used as a marker, apart from bleeding manifestations, of the need to start platelet transfusion, or prophylactic antibiotics for that matter, though literature not always recommends prophylactic antibiotics in such cases. [15] In emergency situations where detailed hematological analysis requires time which a patient doesn't always have, such a marker can prove to be of great value in the timely management of such patients.

\section{Conclusion and Recommendation}

The study results revealed raised temperature to be significantly negatively correlated with total leukocyte count on day 3 only. Furthermore, stratified analysis showed it to be significantly negatively correlated with platelet count on day 3 in patients with longer duration of fever and with total leukocyte count on day 3 in patients aged 40 years or above. Further evaluation of the study findings with a larger sample size is recommended.

\section{Conflict of Interests}

The authors report no conflict of interests.

\section{References}

1. HO. Dengue and Severe Dengue. WHO. 2018.

2. Malavige GN, Fernando S, Fernando DJ, Seneviratne SL. Dengue viral infections. Postgraduate medical journal. 2014; 80(948):588-601. doi. org/10.1136/pgmj.2004.019638

3. Thein S, Aung MM, Shwe TN, Aye M, Zaw A, Aye K et al. Risk factors in dengue shock syndrome. The American journal of tropical medicine and hygiene. 2013; 56 (5):566-72. doi.org/10.4269/ ajtmh.1997.56.566

4. Tang JW, Khanani MR, Zubairi AM, Lam WY, Lai F, Hashmi K et al. A wide spectrum of dengue IgM and PCR positivity post-onset of illness found in a large dengue 3 outbreak in Pakistan. Journal of medical virology. 2008 Dec; 80(12):2113-21.doi: 10.1002/jmv.21290

5. Ashley EA. Dengue fever. Trends in Anaesthesia and Critical Care. 2011; 1(1):39-41. doi:10.1016/j.tacc.2010.10.002

6. Oishi K, Saito M, Mapua CA, Natividad FF. Dengue illness: clinical features and pathogenesis. Journal of infection and chemotherapy. 2007; 13(3):125-33. doi:10.1007/s10156-007-0516-9

7. Pal T, Dutta SK, Mandal S, Saha B, Tripathi A. Differential clinical symptoms among acute phase Indian patients revealed significant association with dengue viral load and serum IFN-gamma level. Journal of Clinical Virology. 2014; 61(3):365-70. doi: 10.1016/j. jcv.2014.09.003

8. Makroo RN, Raina V, Kumar P, Kanth RK. Role of platelet transfusion in the management of dengue patients in a tertiary care hospital. Asian journal of transfusion science. 2007; 1(1):4.doi: 10.4103/09736247.28065 
9. Banerjee A, Paul UK, Bandyopadhyay A. Diagnosis of dengue fever: roles of different laboratory test methods. International Journal of Advances in Medicine. 2018; 5(2):395-9.doi.org/10.18203/23493933

10. Kumar A, Pandit VR, Shetty S, Pattanshetty S, Krish SN, Roy S. A profile of dengue cases admitted to a tertiary care hospital in Karnataka, southern India. Tropical doctor. 2010; 40(1):45-6.

11. Kumar K, Singh PK, Tomar J, Baijal S. Dengue: epidemiology, prevention and pressing need for vaccine development. Asian Pacific Journal of Tropical Medicine. 2010; 3(12):997-1000. doi.org/10.1016/S19957645(11)60017-5

12. Waggoner JJ, Abeynayake J, Sahoo MK, Gresh L, Tellez Y, Gonzalez Ket al. Comparison of the FDA-approved CDC DENV-1-4 Real-Time RT-PCR with a laboratory-developed assay for dengue virus detection and serotyping. Journal of clinical microbiology.2013:51(10):1359-73. doi: 10.1128/JCM.01359-13
13. Khan E, Kisat M, Khan N, Nasir A, Ayub S, Hasan R. Demographic and Clinical Features of Dengue Fever in Pakistan from 2003-2007: A Retrospective Cross-Sectional Study. PLoS ONE. 2010; 5(9). doi: 10.1371/journal.pone.0012505

14. Tanner L, Schreiber M, Low JG, Ong A, Tolfvenstam T, Lai YL et al. Decision Tree Algorithms Predict the Diagnosis and Outcome of Dengue Fever in the Early Phase of Illness. PLoS Neglected Tropical Diseases. 2008; 2(3). doi.org/10.1371/journal.pntd.0000196

15. Thein TL, Lye DC, Leo YS, Wong JG, Hao Y, Wilder-Smith A. Severe neutropenia in dengue patients: prevalence and significance. The American journal of tropical medicine and hygiene. 2014 Jun 4; 90(6):984-7.doi: 10.4269/ajtmh.14-0004 